\title{
Comparison of Pathologist-Detected and Automated Computer-Assisted Image Analysis Detected Sentinel Lymph Node Micrometastases in Breast Cancer
}

\author{
Donald L. Weaver, M.D., David N. Krag, M.D., Edward A. Manna, B.S., Taka Ashikaga, Ph.D., \\ Seth P. Harlow, M.D., Kenneth D. Bauer, Ph.D. \\ University of Vermont, Vermont Cancer Center and Fletcher Allen Health Care, Burlington, Vermont \\ (DLW, DNK, EAM, TA, SPH); and ChromaVision Medical Systems, San Juan Capistrano, California (KDB)
}

Sentinel lymph node biopsy has stimulated interest in identification of micrometastatic disease in lymph nodes, but identifying small clusters of tumor cells or single tumor cells in lymph nodes can be tedious and inaccurate. The optimal method of detecting micrometastases in sentinel nodes has not been established. Detection is dependent on node sectioning strategy and the ability to locate and confirm tumor cells on histologic sections. Immunohistochemical techniques have greatly enhanced detection in histologic sections; however, comparison of detection methodology has not been undertaken. Automated computer-assisted detection of candidate tumor cells may have the potential to significantly assist the pathologist. This study compares computer-assisted micrometastasis detection with routine detection by a pathologist. Cytokeratin-stained sentinel lymph node sections from 100 patients at the University of Vermont were evaluated by automated computer-assisted cell detection. Based on original routine light microscopy screening, 20 cases that were positive and 80 cases that were negative for micrometastases were selected. One-level (43 cases) or two-level (54 cases) cytokeratin-stained sections were examined per lymph node block. All 100 patients had previously been classified as node negative by using routine he-

Copyright (C) 2003 by The United States and Canadian Academy of Pathology, Inc.

VOL. 16, NO. 11, P. 1159, 2003 Printed in the U.S.A.

Date of acceptance: July 14, 2003.

This work was supported by grants (UO1-CA65121, P30-CA22435) from the National Cancer Institute with additional support from ChromaVision Medical Systems.

The data were presented at the 91st annual meeting of the United States and Canadian Academy of Pathology, Chicago, IL, February 27, 2002 and published in abstract format (Mod Pathol 2002; 15:55A-56A).

The views expressed in this article are solely those of the authors and do not necessarily represent the official views of the National Cancer Institute or the U.S. federal government.

Address reprint requests to: Donald L. Weaver, M.D., Department of Pathology, Given E-203, Health Science Complex, University of Vermont College of Medicine, Burlington, VT 05405-0068; fax: 802-656-8892; email: donald.weaver@uvm.edu.

DOI: 10.1097/01.MP.0000092952.21794.AD matoxylin and eosin stained sections. Technical staining problems precluded computer-assisted cell detection scanning in three cases. Computer-assisted cell detection detected 19 of 20 (95.0\%; 95\% confidence interval, $75-100 \%$ ) cases positive by routine light microscopy. Micrometastases missed by computerassisted cell detection were caused by cells outside the instrument's scanning region. Computer-assisted cell detection detected additional micrometastases, undetected by light microscopy, in 8 of 77 (10.4\%; 95\% confidence interval, 5-20\%) cases. The computerassisted cell detection-positive, light microscopymissed detection rate was similar for cases with one (3 of $30 ; 10.0 \%$ ) or two (5 of $47 ; 10.6 \%$ ) cytokeratin sections. Metastases detected by routine light microscopy tended to be larger $(0.01-0.50 \mathrm{~mm})$ than did metastases detected only by computer-assisted cell detection $(0.01-0.03 \mathrm{~mm})$. In a selected series of patients, automated computer-assisted cell detection identified more micrometastases than were identified by routine light microscopy screening of cytokeratin-stained sections. Computer-assisted detection of events that are limited in number or size may be more reliable than detection by a pathologist using routine light microscopy. Factors such as human fatigue, incomplete section screening, and variable staining contribute to missing metastases by routine light microscopy screening. Metastases identified exclusively by computer-assisted cell detection tend to be extremely small, and the clinical significance of their identification is currently unknown.

KEY WORDS: Breast cancer, Cytokeratin, Image analysis, Immunohistochemistry, Micrometastases, Sentinel lymph nodes.

Mod Pathol 2003;16(11):1159-1163

The presence or absence of metastases in the axillary lymph nodes remains the most powerful prognostic factor in breast cancer (1). Because of the high proportion of lymph node-negative patients in 
current practice, sentinel lymph node biopsy is an attractive alternative to full axillary dissection for staging breast cancer. The technique has been shown to be sensitive and specific for predicting the status of the axillary nodes (2-7) and is now under further investigation in two large clinical trials in the United States. Sentinel nodes are often examined more comprehensively than routinely dissected axillary nodes, thus enhancing the identification of small tumor micrometastases. The clinical significance of nodal micrometastases sized $\leq 2.0$ $\mathrm{mm}$ is unclear with respect to overall survival and tumor biology (8-13). Results of prospective trials are still needed to further evaluate the clinical role of micrometastases and isolated tumor cells. The predictive value of occult metastases in sentinel nodes is being evaluated as a correlative study in the National Surgical Adjuvant Breast and Bowel Project B-32 sentinel node trial and the American College of Surgeons Oncology Group Z-0010 study.

Regardless of past conclusions, the reduced number of sentinel nodes has rejuvenated interest in identifying micrometastases. When histologically negative sentinel nodes are examined with additional sections and cytokeratin immunohistochemical stains, the conversion rate to node positive is $10-12 \%$, with one or two immunostains, but increases to $25-30 \%$ if more levels and cytokeratinstained sections are examined $(14,15,16)$. Although not discussed as extensively in the literature, missed metastases (present on review of original slides) have been included under the rubric of occult metastases and have been shown to be present in $2.6 \%$ of sentinel lymph node biopsy cases (16). What has not been discussed is the frequency of missing metastases on immunohistochemistrystained sections. This study was undertaken to test the ability of automated computer-assisted cell detection using image analysis to do the following: (1) detect micrometastases previously detected by a pathologist routinely screening cytokeratin-stained slides and (2) determine the frequency with which computer-assisted cell detection would identify micrometastases not previously detected by pathologist screening.

\section{MATERIALS AND METHODS}

Cytokeratin immunohistochemistry stained lymph node sections from two groups of patients (100 cases) who were participating in sentinel lymph node biopsy research protocols at the University of Vermont were selected for examination by automated computerassisted cell detection. All patients had been previously classified as node negative based on review of hematoxylin and eosin-stained sections of lymph nodes that had been bisected or thinly sliced at 2.0-mm intervals before tissue processing and embedding. Subsequently, deeper sections and cytokeratin stains were performed as part of a research protocol. The first group (43 cases) had one-level cytokeratin-stained sections (LSAB detection kit; DAKO Cytomation, Carpinteria, CA) examined at 0.1 mm deeper into the block. The second group (54 cases) had two-level cytokeratin-stained sections (polymer detection kit; DAKO Cytomation) examined at approximately 0.5 and $1.0 \mathrm{~mm}$ deeper into the block. AE1-AE3 anti-cytokeratin (Chemicon International, Temecula, CA) was used for both groups, and each lymph node block was examined with cytokeratin sections. A pathologist had previously examined all cases with a standard light microscope to determine whether occult micrometastases were present or absent. Cases were selected sequentially in the two series but with a bias toward including cases with pathologist- and light microscope-detected micrometastases ( 20 positive; 80 negative) to test the ability of the automated system to detect known micrometastases. An image analysis system (ChromaVision Automated Cell Imaging System; ChromaVision Medical Systems, Inc., San Juan Capistrano, CA) linked to a computer with automated cell detection software for detecting the chromagen was employed for computer-assisted cell detection. Target antibody was visualized by using diaminobenzidene (3,3'diaminobenzidine), and slides were counterstained with hematoxylin. Slides were loaded into carriers and scanned by the instrument, and images of candidate micrometastases were stored in a computer with a histologic reconstruction image of the scanned area of each slide. Images of candidate tumor cells and cell clusters were presented on the computer screen for pathologist interpretation. Review and classification of the computer-assisted cell detection images of candidate micrometastases was undertaken without knowledge of the light microscopy results. A binomial model was used to calculate $95 \%$ exact confidence intervals. The institutional review board of the University of Vermont approved the research protocol.

\section{RESULTS}

Computer-assisted cell detection identified 19 of $20(95.0 \%$; 95\% confidence interval $=75$ to $100 \%)$ cases with micrometastases that were originally detected by pathologist review of the cytokeratinstained sections using only light microscopy. The single case in which micrometastases were missed by computer-assisted cell detection was due to cells being present outside the physical limits of slide scanning for the instrument (Fig. 1). Cytokeratinpositive debris in excess of the preset software storage capacity settings precluded scanning of some 


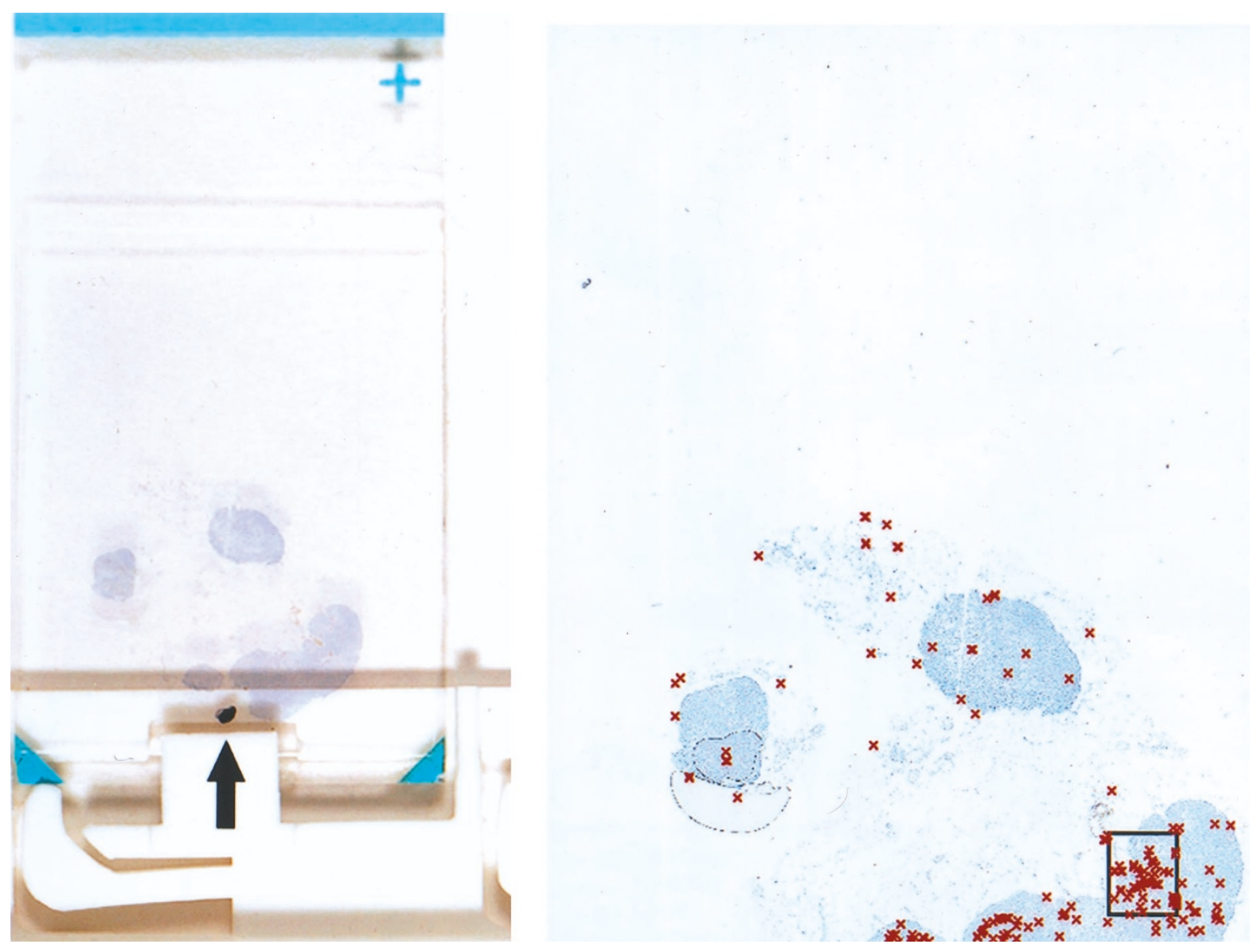

FIGURE 1. Example of micrometastasis missed by computer-assisted cell detection but detected by pathologist. Left side demonstrates the microscope slide in the plastic slide carrier. The lower portion of the lymph node was not scanned because the section was mounted too low on the glass slide, was out of the scan range of the instrument, and was obscured by the supporting slide carrier. The black arrow and dot indicate the position of the micrometastasis. Right side demonstrates the computer-reconstructed image of the glass slide, confirming incomplete scanning of the lymph node.

slides, and three cases negative for micrometastases by pathologist review with light microscopy were unsuitable for analysis because of excessive debris. Computer-assisted cell detection detected additional micrometastases, initially undetected by pathologist review with light microscopy, in 8 of 77 (10.4\%; $95 \%$ confidence interval $=5$ to $20 \%$ ) cases. The computer-assisted cell detection-positive, light microscopy-missed detection rate was similar for cases with one (3 of $30 ; 10.0 \%$ ) or two (5 of 47 ; $10.6 \%)$ cytokeratin sections. The range in size of metastases detected by a pathologist using only light microscopy tended to be larger (0.01-0.50 $\mathrm{mm}$ ) than that of metastases first detected by computer-assisted cell detection (0.01-0.03 mm). Examples of computer-assisted cell detection-detected candidate metastases and debris are presented in Fig. 2.

\section{DISCUSSION}

This study examined both the ability of an automated computer-assisted cell detection system to locate metastases previously detected by pathologist review of cytokeratin-stained sections and the ability to detect metastases not previously identified by pathologist review. The automated computer-assisted cell detection system detected all previously identified metastases that were within the scanning region of the slides. Slides had been manufactured before initiation of the study, and therefore some metastases (one case) were undetectable to the instrument because they were outside the scan area.

Cytokeratin immunohistochemistry can enhance detection of micrometastases; however, as in examination of routinely stained slides, metastases can still be missed. Factors contributing to missed metastases include the following: small metastasis size, variable cell staining, incomplete screening of sections, and human fatigue. In this study, micrometastases not previously detected by pathologist screening of cytokeratin-stained sections were identified by automated computer-assisted cell detection, and $10 \%$ of cases classified as negative by initial pathologist review had metastases detected. 

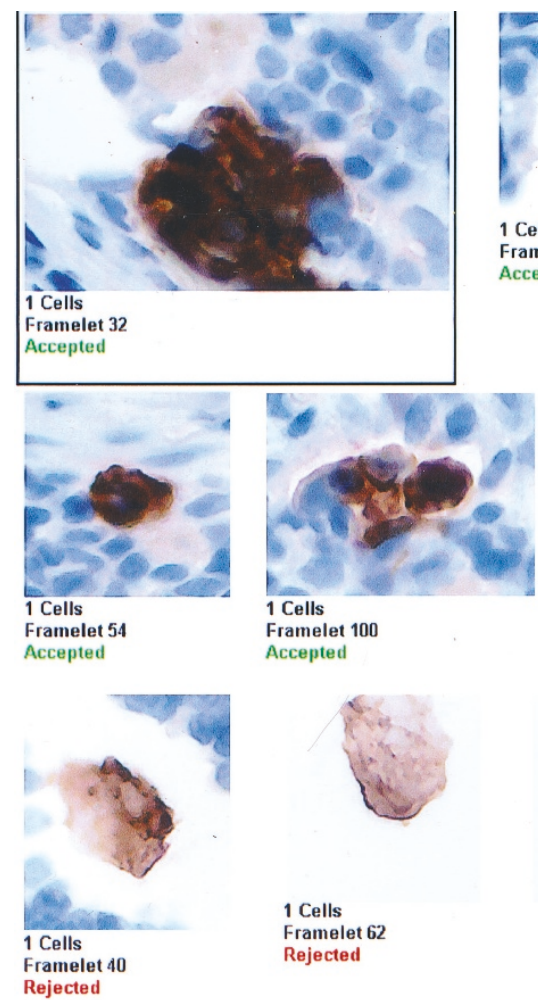
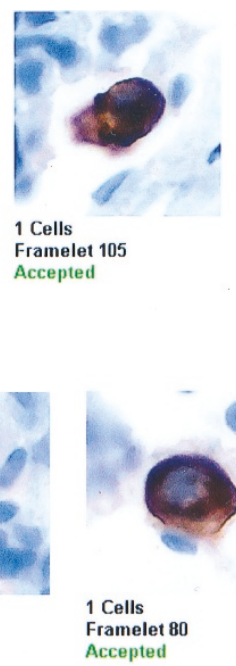

Accepted

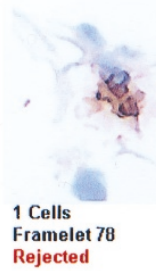

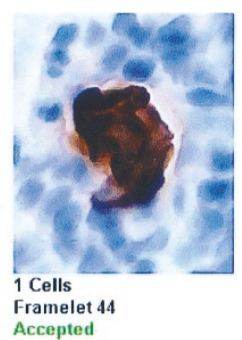

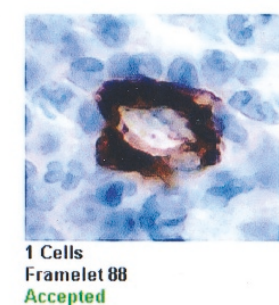

Accepted

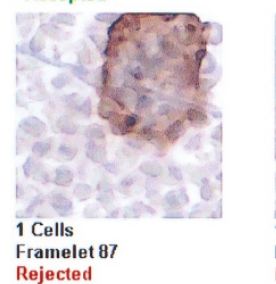

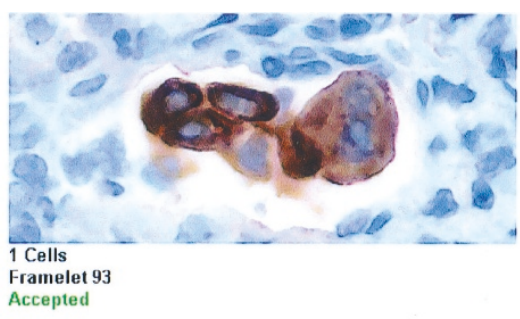

Accepted

FIGURE 2. Individual stored images demonstrating candidate metastases identified by an automated image analysis-based computer-assisted cell detection system, then categorized by a pathologist. Rejected candidate images are frequently exfoliated cuticular debris that contaminates the slide before staining.

Missed metastases were usually due to single cells or two- to three-cell clusters that were faintly stained and present in low number on the slides. Under the recently revised sixth edition of the AJCC staging manual, these missed occult metastases fall below the lower limit of micrometastases and would qualify as isolated tumor cells or tumor cell clusters (ITCs) (17). ITCs are a new category of metastases in which no metastasis is $>0.2 \mathrm{~mm}$, and they are classified as pN0. All metastases, once identified by computer-assisted cell detection, could be identified by human visual inspection of the stained sections using standard light microscopy. A few missed metastases were more obvious and may have been missed through human screening error caused by fatigue or by incomplete slide scanning.

The detection of micrometastases and ITCs in lymph nodes has practical and economic limitations (18). As discussed in this article, identification of micrometastases is dependent on the ability of the pathologist to reliably detect tumor cells on the slides. Cytokeratin immunohistochemistry and computer-assisted cell detection can enhance detection. However, micrometastasis identification is also highly dependent on the lymph node sectioning interval. Any identified metastasis that is smaller than the node-sectioning interval may have been identified by chance and could just as easily have been missed. For example, to reliably detect two- to three-cell clusters $(0.02-\mathrm{mm}$ metastases), lymph nodes must be entirely embedded and sectioned at $20-\mu \mathrm{m}$ intervals. This strategy would require 100 sections per lymph node block if nodes were cut at $2.0-\mathrm{mm}$ intervals before embedding. Production of large numbers of immunohistochemically stained slides is costly, and review of the slides is time consuming, regardless of whether sections are screened primarily by pathologists using a light microscope or computer-assisted cell detection. In addition, reimbursement for multiple cytokeratin stains on a single specimen has not been resolved. The time to scan each slide with automated computer-assisted cell detection was approximately 30-40 minutes with the technology available at the time of this study. Any candidate metastases detected by computer-assisted cell detection must be confirmed by pathologist review of the digital images and/or a re-evaluation of the slide. Although this study demonstrates that pathologist review of cytokeratin-stained sections will miss $\geq 10 \%$ of the metastases present on the slides, it does not address the potential metastases remaining in the paraffin tissue block or in tissue removed but not examined. When evaluating the clinical difference between patients with and without micrometastases identified, pathologists and clinicians must also consider the probability that 
the metastasis could have been missed. In other words, a pathologist would not be able to confidently ensure a clinician, based on statistical principles, that a patient with a negative sentinel node evaluated with one or two cytokeratin-stained sections, as performed in this study, is categorically different from a patient with a two- or three-cell cluster identified. It is therefore critical that pathologists document the size and magnitude of micrometastases identified (19).

The automated computer-assisted cell detection system used in this study has a user-defined maximum number of images per slide that are stored for later interpretation by a trained individual. For the slides stained with the polymer detection kit, a setting of 500 events was more than sufficient for capture of all candidate metastases. For the slides stained with the LSAB detection kit, a setting of 1000 events was needed. Some slides contained excessive cytokeratin-stained debris and, although possible, it was not practical to increase the stored events to $>1000$ during this study. Prospective reduction of extraneous cytokeratin positive debris is desirable if image analysis-based computerassisted cell detection is to be performed.

Automated computer-assisted cell detection can assist in detecting occult metastases. This study has demonstrated that an automated computerassisted cell detection system is proficient at identifying micrometastases detected by human screening and can identify additional small micrometastases (isolated tumor cells and tumor cell clusters) that may be missed by human visual screening of cytokeratin-stained slides. The findings also underscore the false sense of security created by examination of cytokeratin-stained slides and establishes a baseline for the proportion and magnitude of occult metastases that are missed by a pathologist screening cytokeratin-stained sections.

\section{REFERENCES}

1. Fleming ID, Cooper JS, Henson DE, Hutter RVP, Kennedy BJ, Murphy GP, et al., editors. AJCC cancer staging manual. 5th ed. New York: Lippincott-Raven; 1997. p. 171-80.

2. Albertini JJ, Lyman GH, Cox C, Yeatman T, Balducci L, Ku N, et al. Lymphatic mapping and sentinel node biopsy in the patient with breast cancer. JAMA 1996;276:1818-22.

3. Giuliano AE, Kirgan DM, Guenther JM, Morton DL. Lymphatic mapping and sentinel lymphadenectomy for breast cancer. Ann Surg 1994;220:391-401.
4. Hill AD, Tran K, Akhurst T, Yeung H, Yeh SD, Rosen PP, et al. Lessons learned from 500 cases of lymphatic mapping for breast cancer. Ann Surg 1999;229:528-35.

5. Kollias J, Gill PG, Chatterton BE, Hall VE, Bochner MA, Coventry BJ, et al. Reliability of sentinel node status in predicting axillary lymph node involvement in breast cancer. Med J Aust 1999;171:461-5.

6. Krag D, Weaver D, Ashikaga T, Moffat F, Klimberg S, Shriver $\mathrm{C}$, et al. The sentinel node in breast cancer: a multicenter validation study. N Engl J Med 1998;339:941-6.

7. Veronesi U, Paganelli G, Galimberti V, Viale G, Zurrida S, Bedoni M, et al. Sentinel-node biopsy to avoid axillary dissection in breast cancer with clinically negative lymph nodes. Lancet 1997;349:1864-7.

8. Fisher E, Palekar A, Rockette H, Redmond C, Fisher B. Pathologic findings from the National Surgical Adjuvant Breast Project (protocol no. 4): V. Significance of axillary nodal micro- and macrometastases. Cancer 1978;42:2032-8.

9. Huvos AG, Hutter RVP, Berg JW. Significance of axillary macrometastases and micrometastases in mammary cancer. Ann Surg 1971;173:44-6.

10. Colpaert C, Vermeulen P, Jeuris W, van Beest P, Goovaerts G, Weyler J, et al. Early distant relapse in "node negative" breast cancer patients is not predicted by occult axillary lymph node metastases, but by the features of the primary tumour. J Pathol 2001;193:442-9.

11. Hansen NM, Grube BJ, Te W, Brennan ML, Turner R, Guiliano AE. Clinical significance of axillary micrometastases in breast cancer: how small is too small [abstract 91]? Proc ASCO 2001;20:24a.

12. Cote RJ, Peterson HF, Chaiwun B, Gelber RD, Goldhirsch A, Castiglione-Gertsch M, et al. Role of immunohistochemical detection of lymph-node metastases in management of breast cancer. International Breast Cancer Study Group. Lancet 1999;354(9182):896-900.

13. Nasser IA, Lee AKC, Bosari S, Saganich R, Heatley G, Silverman ML. Occult axillary lymph node metastases in "nodenegative" breast carcinoma. Hum Pathol 1993;24:950-7.

14. Dowlatshahi K, Fan M, Anderson JM, Bloom KJ. Occult metastases in sentinel nodes of 200 patients with operable breast cancer. Ann Surg Oncol 2001;8:675-82.

15. Turner RR, Ollila DW, Stern S, Giuliano AE. Optimal histopathologic examination of the sentinel lymph node for breast carcinoma staging. Am J Surg Pathol 1999;23:263-7.

16. Weaver DL, Krag DN, Ashikaga T, Harlow SP, O'Connell M. Pathologic analysis of sentinel and non-sentinel lymph nodes in breast carcinoma: a multicenter study. Cancer 2000;88:1099-107.

17. Greene FL, Page DL, Fleming ID, Fritz AG, Balch CM, Haller DG, et al., editors. AJCC Cancer Staging Manual. 6th ed. New York: Springer; 2002.

18. Weaver DL. Sentinel lymph nodes and breast carcinoma: which micrometastases are clinically significant? Am J Surg Pathol 2003;27:842-5.

19. Fitzgibbons PL, Page DL, Weaver D, Thor AD, Allred DC, Clark GM, et al. Prognostic factors in breast cancer: College of American Pathologists consensus statement 1999. Arch Pathol Lab Med 2000;124:966-78. 